Int. J. Dev. Biol. 58: 35-43 (2014)

doi: $10.1387 / \mathrm{ijdb} .130305 \mathrm{md}$

\title{
Focal adhesion kinase as a mechanotransducer during rapid brain growth of the chick embryo
}

\author{
MARY E. DESMOND ${ }^{1}$, JANICE E. KNEPPER ${ }^{1}$, ANGELA J. DIBENEDETTO ${ }^{1}$, ELIZABETH MALAUGH ${ }^{1}$, \\ SAGRARIO CALLEJO², RAQUEL CARRETERO'², MARIA-ISABEL ALONSO ${ }^{2}$ and ANGEL GATO² \\ ${ }^{1}$ Department of Biology, Villanova University, Villanova, PA, USA and ${ }^{2}$ Departamento de Anatomia y Radiologia, \\ Facultad de Medicina, Universidad de Valladolid, Valladolid, Spain
}

\begin{abstract}
Expansion of the hollow fluid-filled embryonic brain occurs by an increase in intraluminal pressure created by accumulation of cerebrospinal fluid (CSF). Experiments have shown a direct correlation between cavity pressure and cell proliferation within the neuroepithelium. These findings lead us to ask how mechanistically this might come about. Are there perhaps molecules on the luminal surface of the embryonic neuroepithelium, such as focal adhesion kinases (FAKs) known to respond to tension in other epithelial cells? Immunodetection using antibodies to total FAK and p-FAK was performed with subsequent confocal analysis of the pattern of their activation under normal intraluminal pressure and induced chronic pressure. Western analysis was also done to look at the amount of FAK expression, as well as its activation under these same conditions. Using immunolocalization, we have shown that FAK is present and activated on both apical and basolateral surfaces and within the cytoplasm of the neuroepithelial cells. This pattern changed profoundly when the neuroepithelium was under pressure. By Western blot, we have shown that FAK was upregulated and activated in the neuroepithelium of the embryos just after the neural tube becomes a closed pressurized system, with phosphorylation detected on the luminal instead of the basal surface, along with an increase in cell proliferation. Chronic hyper-pressure does not induce an increase in phosphorylation of FAK. In conclusion, here we show that neuroepithelial cells respond to intraluminal pressure via FAK phosphorylation on the luminal surface.
\end{abstract}

KEY WORDS: cerebrospinal fluid, neuroepithelium, mitosis, hydrostatic pressure, FAK, cell proliferation

\section{Introduction}

The vertebrate embryonic brain is the first organ to develop and does so as a hollow structure similar to a balloon that expands rapidly during early embryogenesis. This growth occurs as an active, interdependent synergism between the increase of cerebrospinal fluid (CSF) within the cavity and proliferation of the cells comprising the neuroepithelium (Gato \& Desmond, 2009). Initially, the embryonic brain is an open tube that becomes sequentially sealed anteriorly and posteriorly by closure of the anterior neuropore and occlusion of the spinal neurocoel (Desmond \& Schoenwolf 1984). As the tube is closed it also forms three vesicles anterior to posterior: the prosencepalon/forebrain, the mesencephalon/midbrain and the rhombencephalon/hindbrain. Expansion of the brain now rapidly occurs regulated by an increase in intraluminal pressure created by an increase in CSF (Desmond \& Jacobson, 1977; Pacheco et al., 1986; Desmond \& Levitan, 2002). At the same time that the brain expands, the neuroepithelium undergoes rapid proliferation and subsequent differentiation. The cells lining the lumen are germinal neurons that proliferate to form the outer layers. Cells of the inner layer also migrate to the outer layers and begin to differentiate into intermediate and terminal neurons.

Accumulation of CSF via an osmotic gradient not only creates internal pressure (Desmond \& Jacobson, 1977; Gato et al., 1993; Desmond et al., 2005) but also supplies trophic factors such as FGF-2 for the neuroepithelium (Gato et al., 2005; Martin et al., 2006; Parada, et al., 2008). A decrease in the intraluminal pressure of the chick embryonic brain results in $50 \%$ reduction in both the tissue volume and cell number (Desmond 1985; Desmond \& Jacobson 1977). Conversely, an increase in the intraluminal pressure for one hour elicits a two-fold increase in the number of cells within the neuroepithelium (Desmond et al., 2005). These findings

Abbreviations used in this paper: CSF, cerebrospinal fluid; FAK, focal adhesion kinase.

\footnotetext{
*Address correspondence to: Mary E. Desmond. Department of Biology Villanova University Villanova, PA 19010, USA. Tel: +1-610-519-4829. Fax: +1-610-519-7783. E-mail: Mary.Desmond@Villanova.edu
} 
suggest that intraluminal pressure regulates cell proliferation which immediately leads us to ask how mechanistically this might come about. Are there perhaps molecules on the luminal surface of the embryonic neuroepithelium that act as mechanotransducers such as those found on the luminal surface of blood vessels (Lehoux et al., 2003, 2005)?

These mechanotransducers or focal adhesion kinases (FAKs) have been shown to increase on the apical surface of segments of perfused rabbit aorta with doubling of the internal pressure of the segments (Lehoux, et al., 2005). They have also been shown to be localized on the apical surface of retinal pigrmented epithelium during phagocytosis (Finneman 2003); the inner hair cells of the utricle of the inner ear (Evans \& Muller 2000); and the neointima of new blood vessels (Cai et al., 2009). Other studies show them to be upregulated in cultures of lung epithelial cells under strain correlated with an increase in cell proliferation, with Src and ERK activation (Chaturvedi, et al., 2007). The localization of FAKs was not elucidated by the lung researchers. Most likely these FAKS are on the basal surface of the lung epithelium as they are in most epithelia other than those surrounding fluid filled cavities such as blood vessels and the embryonic brain reported here in our study. In fact, much more is known about FAK expression on the basal surface compared with its expression of the apical surface. Nonetheless, there is ample evidence of apical expression in blood vessels and other tissues.

Since the pressure being applied to the neuroepithelium is also steadily applied, we hypothesized that the mechano-detection pathway in the neuroepithelium may be one initiated by the phosphorylation of FAK. The embryonic brain is primarily a fluid filled cavity and thus similar to the aorta except for the difference in the magnitude of the intra-luminal pressure. In fact, the intraluminal pressure within the aorta is at least 23 times more than that within the embryonic brain based on the measurements of static pressure within the aorta and the embryonic brain. The internal static pressure in the aorta is $80 \mathrm{~mm} \mathrm{Hg}$ (Lehoux, et al., 2005) compared with the average static pressure of $3.5 \mathrm{~mm} \mathrm{Hg}$ of the CSF within the embryonic brain vesicles (Desmond, et al., 2005). This great difference in magnitude of internal pressure, may mean that the mechano-receptors on the surface of the aorta are more primed to respond to changes in pressure than they are in the embryonic brain. Thus, we predicted that there would be a change in the phosphorylation of FAKs on the surfaces of the neuroepithelium in response to an increase in intraluminal pressure but that the response may not be as pronounced as in the aortic endothelium.

FAK is a protein kinase that forms a focal complex on the cytoplasmic side of the cell membrane and autophosphorylates in response to integrin signaling (Katsumi et al., 2004; Mitra et al., 2005). It is a member of the nonreceptor tyrosine protein kinase (PTK) subfamily and mediates bidirectional signaling with either the extra-cellular matrix (Swartz, 2001) on the basal surface or extra-cellular fluid such as blood in vessels on the luminal surface as described above.

Autophosphorylation, and hence activation of FAK occurs when integrin-binding proteins such as paxillin and talin are recruited to an active integrin site at the cell surface. FAK as a protein tyrosine kinase donates a phosphate group to other proteins to activate or deactivate them. FAK itself can be phosphorylated to initiate active intracellular signaling cascades. Upon integrin signaling, FAK migrates to the site of focal adhesions where it can self-activate by forming a dimer with another FAK protein, a process called auto-or trans-phosphorylation. Once FAK is activated it recruits and subsequently phosphorylates other intracellular proteins such as Src and Grb2 to initiate various signaling cascades within the cell (Zhao and Guan, 2011). Src and ERK 1/2 have been identified as downstream signaling molecules of FAK which are activated upon cellular strain to induce cell proliferation (Chaturvedi, et al., 2007). Most of what is known about the FAK signaling pathway is for FAK on the basal surface.

It is our hypothesis that increased pressure generated by the CSF upon the neuroepithelium is detected by FAK on its luminal surface which in turn transmits this signal to the nucleus via the Src-dependent ERK1/2 cascade (Cho et al., 2005; Lehoux, et al., 2005). In this study we focused only on whether FAK was indeed on the luminal surface as shown for blood vessels.

Based on the present understanding of FAK, and our hypothesis that intraluminal pressure gives a positive proliferative signal that is transmitted by FAK, we set out to test several predictions focused on establishing that FAK is indeed on the luminal surface of the neuroepithelium of early fluid-filled embryonic brains and responds to changes in internal fluid pressure. First, we planned to immunologically localize FAK on the apical and basal surfaces of the neuroepithelium at three phases of embryonic brain growth: the period immediately preceding neural tube closure, just after closure, and the period of rapid brain enlargement following closure. Secondly, we set out to immunologically assess phosphorylation of FAK at two tyrosine residues, tyr 397 and tyr 861. Finally, we sought to demonstrate a difference in the amount of FAK and its activation state on the luminal and basal epithelial surfaces in brains in which the internal pressure had been increased.

\section{Results}

To place the immunolocalization of FAK and p-FAK in context, it is important to recall that the neuroepithelium is initially pseudostratified and then truly stratified varying in the number of cell layers spanning the region between the two surfaces. The actual number of layers increases with the embryonic stage and differs for the three initial embryonic regions. As differentiation proceeds, neurons migrate different distances away from the basal surface and some neurons have axons that extend to the apical surface making a nuclear free layer. The outer one- sixth of the neuroepithelium, i.e. the basal surface, is mostly free of cell bodies/nuclei and rich in axonal extensions plus extra-cellular matrix. The inner luminal or apical surface is in contact with CSF while the basal surface is in contact with the extra-cellular matrix.

\section{Immunolocalization of FAKs during period of brain enlargement}

We examined the localization of FAK and p-FAK in the neuroepithelium at stages of development immediately prior to and during the period of early brain growth (stages $\mathrm{HH} 8-24$ ). We found label in the neuroepithelium for all three brain vesicles in all of the stages examined but focused our attention on the midbrain since we have abundant information on the cellular dynamics of the neuroepithelium of the midbrain.

As illustrated in Fig. 1, the pattern of localization for FAK was concentrated in three distinct regions: at the basal surface, at the luminal surface, and across the entire neuroepithelium within the cytoplasm of cells (Fig. 1A). FAK at the basal surface occurred as a prominent band equal to a sixth of the total width of the neuroepithelium whereas at the luminal surface it was a distinct thin band 

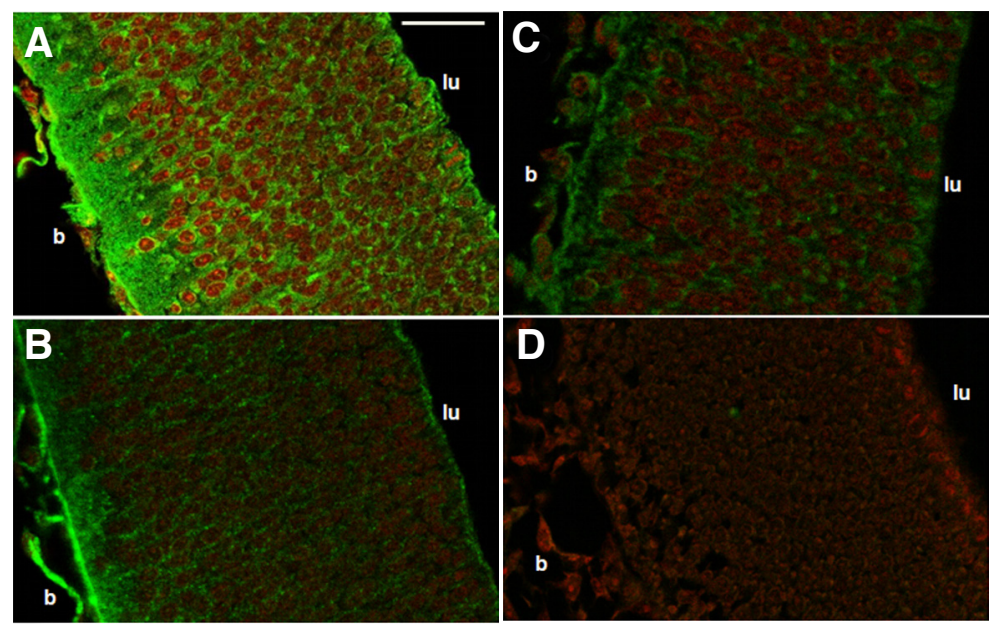

Fig. 1. Immunodetection of pFAK on mesencephlons of stage $\mathbf{2 0}$ embryos. Staining shows FAK (A) and p-FAK (B,C) and a negative control for p-FAK 397 (D) using antibodies for different tyrosine residues, 861 (B) and 397 (C). Both forms of FAK exhibit green fluorescence and the nuclei are stained red with propidium iodide. Note that the localization of FAK is very abundant in the cytoplasm of cells across the entire neuroepithelium as well as exhibiting a wide band at the basal surface (b) on the left and a pronounced band at the luminal surface (I). p-FAK is also localized at both the basal and luminal surfaces and in the cytoplasm of the cells in between but there is less intense cytoplasmic staining of the neuroepithelial cells for $p$-FAK compared to FAK. The staining patterns for $p$-FAK are similar for the two antibodies, tyrosine 397 and 861. There seems to be more p-FAK localization in the cytoplasm using p-FAK specific for tyrosine 397 however less staining at the surface. The magnification bar equals $25 \mathrm{um}$, thus these photomicrographs are magnified 280X.

much narrower in width than the basal label. In the region between the two surfaces, FAK was intensely labeled in the cytoplasm of the cells resembling a cross section of pimento inside stacked green olives (Fig. 1A). The immunolabeling of p-FAK when compared with that of the FAK pattern showed a similar distribution but was less intense overall and more prominent at the surfaces (Fig. 1B, C). The band of p-FAK immunolabel at the basal surface is thinner than that of the FAK. The labeling of the basal surface appeared more uniform than that of the luminal surface. The band of $p$-FAK on the luminal surface also appeared more uniform than that of FAK. The cytoplasmic labeling is much fainter than that of FAK. Most importantly, the pattern of FAK activation was the same for both antibodies used, namely, that at tyr 861 (Fig. 1B) and the one at tyr 397 (Fig. 1C) but the signal was more intense for the antibody directed against tyr 861 . Fig. 1D represents a negative with no primary antibody.

FAK activation is apparent by immunodetection early in development and in all three brain vesicles. Fig. 2, A-C illustrates p-FAK localization in the forebrain $(A)$ and midbrain $(B, C)$. Interestingly, $\mathrm{p}$-FAK is present on the luminal and basal surfaces while the tubular brain is still open to the outside and thus unable to accumulate CSF to form intraluminal pressure (see Fig. 2A of the prosencephalon of a stage $\mathrm{HH} 8$ embryo with the anterior neuropore (asterisk) clearly in view). The photomicrographs in Figs. 2 B,C are from the embryonic mesencephalons at $\mathrm{HH}$ stages 14 and 18 in which the brain is a completely closed tube. Although the images are at different magnifications compared with $2 \mathrm{~A}, \mathrm{p}-\mathrm{FAK}$ is clearly visible on the surfaces as well as across the neuroepithelium. This distribution of $p$-FAK immunolabeling is especially apparent at the higher magnification view in Fig. 1 B,C.

Although p-FAK is present early in development, it increases in intensity in the neuroepithelia of older stage embryos (compare $2 \mathrm{C}$ with $2 \mathrm{~A}$ ) taken at identical exposures. In contrast, the intensity of immunolabel of $p$-FAK labeling is less distinct in the posterior region of the neural tube compared with the anterior (image not shown). This is consistent with the fact that formation of the actual tubular brain of the chick begins in the most anterior region, the prosencephalon, and proceeds posteriorly.

In addition to noting the pattern of $\mathrm{p}-\mathrm{FAK}$ and FAK in the embryonic brain, we observed $p-F A K$ in the lens, cranial mesenchyme, endothelium of blood vessels, endocardium, cranial ganglia and inter-somitic furrow (data not shown), as others have previously reported (Hens and Desimone, 1995; Kokkinos et al., 2007).

When comparing the activation pattern of FAK with total FAK, it appears that FAK is differentially activated at the membrane edges while total FAK also exhibits intense internal cytoplasmic labeling. This suggests that activation of FAK occurs mostly on the surfaces which one might expect due to interaction with integrin complexes.

\section{Western blot analysis of FAK phosphorylation process in neuroepithelium}

Western blot analysis shows that FAK is present and activated in the neuroepithelium at $\mathrm{HH}$ stage 10 at low levels and increases in intensity in $\mathrm{HH}$ stages 12 to 24 (Fig. $3 \mathrm{~A}, \mathrm{~B}$ ). Expression at stage 10 appears to be very low; however, because of the microscopic volume of tissue from each embryo
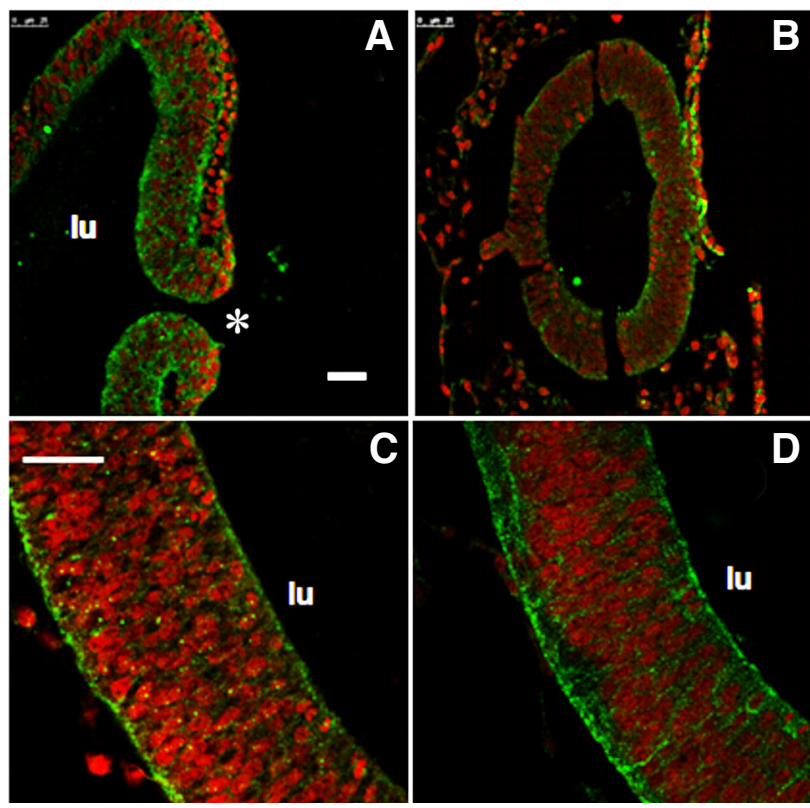

Fig. 2. Photomicrographs of representative sections (HH 8,14,18) of the forebrain $(A)$ and midbrain $(B, C)$ of the embryonic neural tube just prior to and during rapid brain growth showing that $p-F A K$, tyr 861 (green) is on both of the surfaces, basal and apical, of the embryonic neuroepithelium prior to complete fusion of the anterior neuropore $(*)$. Localization of p-FAK on the surfaces continues once the neural tube is completely closed and throughout brain expansion (B, C, stages HH 14,18) increasing to levels shown in Fig. 1 (stage HH 20). The magnification bar equals $25 \mu \mathrm{m}$ thus these photomicrographs are magnified 600 times. 
at that stage precluded loading equal amounts of protein on the gel. After the intraluminal pressure increases at $\mathrm{HH}$ stage 11, there is strong expression of both total and functional (phosphorylated) forms of FAK. We compared the intensity of FAK and p-FAK relative to the control GADPH (Fig. 3B) at the selected embryonic stages, shown as percent of the maximum relative expression. The maximum relative expression of FAK is at stage 12 just after the neural tube is under pressure and then drops precipitously at stage 14 remaining at a low level to stage 24 . More importantly, once the neural tube is under pressure after stage 11, p-FAK is high at stage $12(74 \%)$ increases to maximum at stage 14 then drops back down to $88 \%$ at stage 18 and levels off to $32 \%$ at stage 24. Importantly, although the total FAK became reduced in relative amount at stages 14 and 18, a higher proportion of the kinase was in the active phosphorylated form.

\section{The effects of intraluminal pressure on p-FAK and FAK ex- pression patterns}

In order to test the hypothesis that an increase in intraluminal

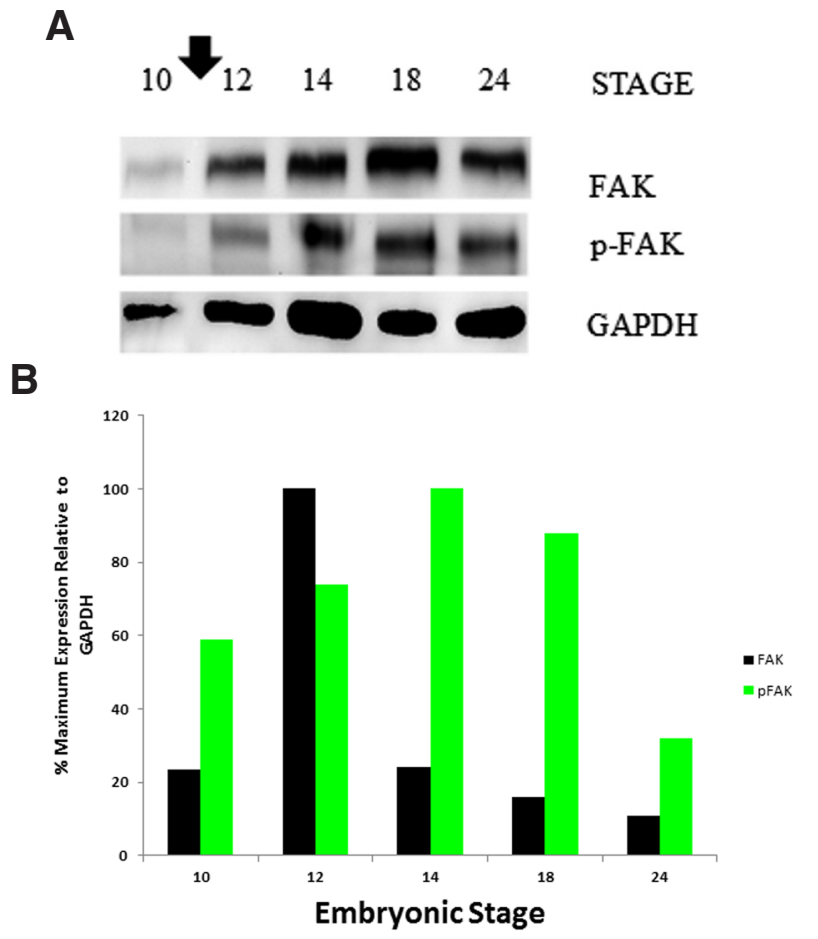

Fig. 3. Quantitative determination of developmental profile of FAK expression and activation. (A) A Western blot of homogenates of neuroepithelia from whole brains at selected embryonic stages (10-24) showing that both FAK and p-FAK increase greatly once the neuroepithelium is under physiological pressure. The bold arrow between stage 10 and 12 indicates the time in development when pressure increases due to the closure of the neural tube by occlusion of the spinal neurocoel at stage $\mathrm{HH}$ 11. FAK was detected at an apparent molecular weight of $120 \mathrm{kd}$ while the phosphorylated form ran slightly larger at $125 \mathrm{kd}$. GAPDH migrated at 38 $k d$. (B) A comparison of the densities for p-FAK and FAK shown in the gel in Figure 3 for the embryonic stages shown. The densities are expressed as a percent of maximum expression relative to GAPDH. The total amount of FAK appears to peak at stage 12 then greatly decreases as it is distributed among more cells with advancing development. The percent of maximum expression of p-FAK remains over $75 \%$ in the neuroepithelium until it drops significantly to $32 \%$ at stage 24 .

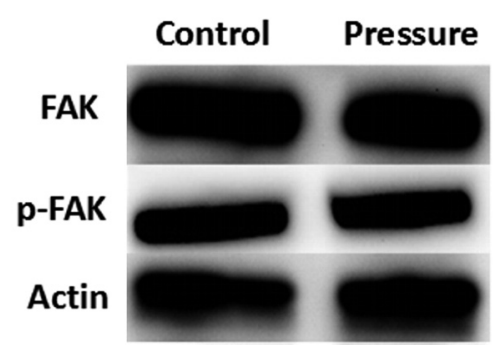

Fig. 4. A western blot of FAK and p-FAK from homogenized neuroepithelium of control brains and brains subjected to hyper-pressure. The samples on the left column are from control brains under normal physiological pressure while those on the right column are from brains under chronic hyper-pressure. There appears to be no difference in expression of either the total or phosphorylated FAK within the neuroepithelium when under chronic hyper-pressure.

pressure initiates an increase in p-FAK, we compared FAK with p-FAK expression within the neuroepithelium after experimentally increasing the intraluminal pressure. Analysis of the western blot shown in Fig. 4 reveals no apparent difference between the expression of FAK or its activation after a chronic increase in pressure. Since there was no apparent change in FAKs within the neuroepithelium overall, we analyzed the intensity of FAK and p-FAK labeling at both the basal and luminal surfaces to see if the level of FAK expression or activation had changed as a consequence of increased pressure. As shown in Fig. $5 \mathrm{~A}-\mathrm{F}$ there were no significant changes in FAK expression on either the basal or luminal surface. However, when we analyzed phosphorylated FAK on the surfaces of the neuroepithelium, we found significant changes for both the basal and luminal surfaces (Fig. 5 G-L). There was a $50 \%$ reduction of $\mathrm{p}-\mathrm{FAK}$ on the basal surface as opposed to a $26 \%$ increase of p-FAK on the luminal surface after an increase in intraluminal pressure. Both of these changes were statistically significant using a Student's t-test. The quantitative results (western analysis and immunofluorescence quantitation) and the qualitative results (high density expression along the edges), suggests that an increase in pressure induces activation of FAK on the luminal surface. This supports the idea that there is a relation between intraluminal pressure and neuroepithelial FAK activity.

In order to know if the change in localization of FAK expression induced by an increase in intraluminal pressure effected cell proliferation, we compared the number of cells expressing PCNA (Proliferating Cell Nuclear Antigen) within the neuroepithelium of brains under induced hyper-pressure with controls (Fig. 6). There was nearly a $17 \%$ increase in the number of PCNA positive cells of the neuroepithelium in brains under pressure. This suggests that chronic increased pressure via BDX (4-nitrophenyl $-\beta-D$ xylopyranoside) increases the number of cells in the DNA synthesis stage of the cell cycle. However to rule out the fact that BDX may induce non- pressure related changes within the neuroepithelium, we cultured neurepithelium in presence of CSF from both control and BDX treated embryos and found no changes in cell mitotic activity (data not shown).

\section{Discussion}

A major finding of this study is that FAK, as well as its activated form, was immunolocalized for the first time on the neuroepithelium during the period of brain growth driven by internal pressure. FAK, 
a mechanotransducer in other physiological systems, is known to detect pressure and transmit signals to the nucleus via the src-Erk cascade. More importantly, we showed that an increase in intraluminal pressure elicited a significant increase of $p$-FAK on the luminal surface of the mesencephalon with corresponding decreased levels at the basal surface (Fig. 6). Our findings taken together suggest that an increase in intraluminal pressure results in a different pattern of FAK activation from basal to luminal surfaces which presumably facilitates the transduction of signals that ultimately result in a proliferative response in the neuroepithelium. Our results support the hypothesis that a neuroepithelial receptor system to intra-luminal pressure is working during early development to accommodate brain cavity and neuroepithelial wall growth.

\section{FAK expression in the neuroepithelium}

FAK has been described previously in the vertebrate embryonic neuroepithelium (Hens \& DeSimone, 1995) but our results are the first to show that FAKs exhibit a specific pattern of localization within the neuroepithelium especially on the surfaces. Putting our results in the context of early brain growth, an increase in the volume of the cavity is linked with an increase in the size of the neuroepithelium. Both increases in size are related. Cavity volume increases by a
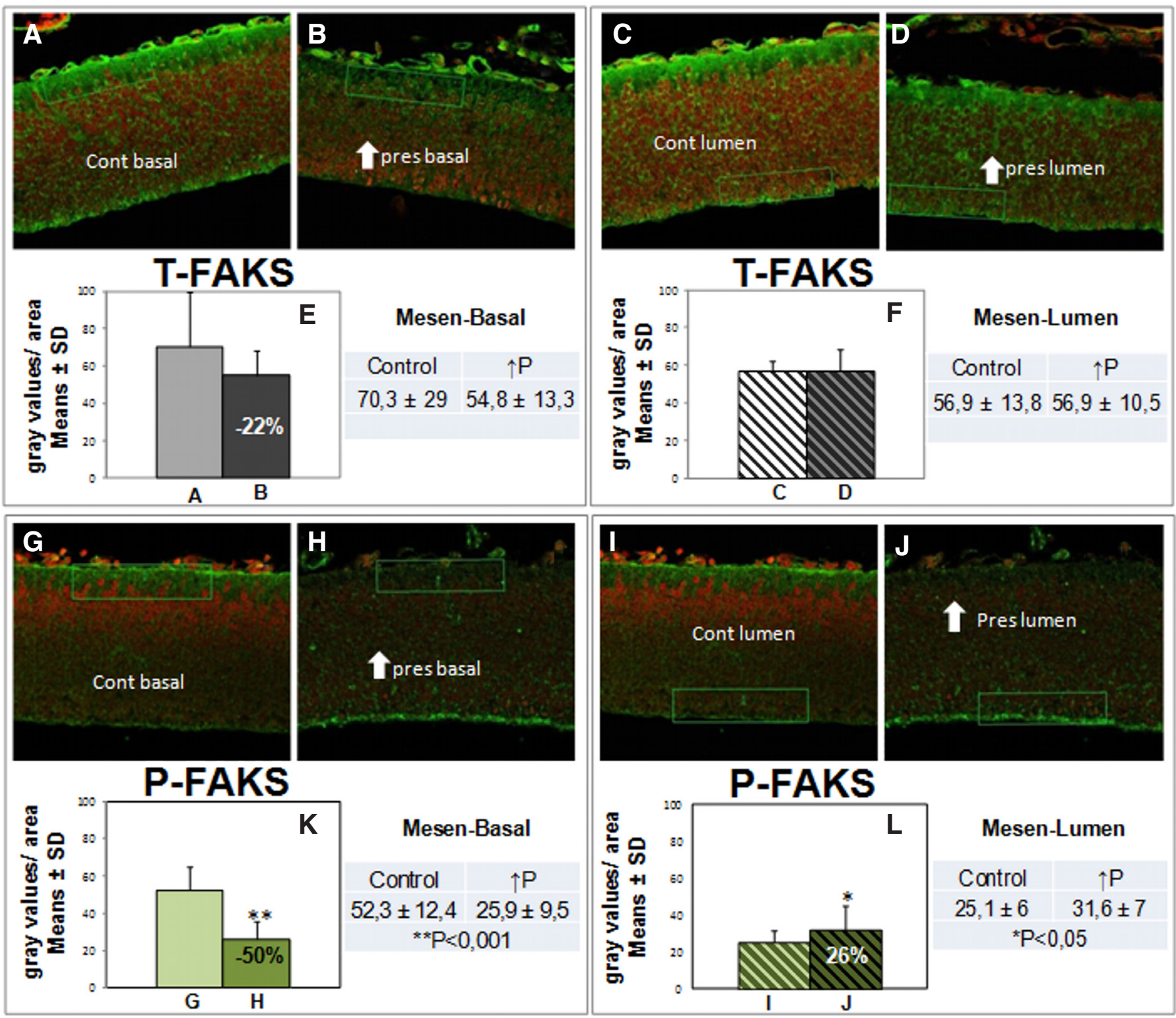

Fig. 5. Localization of FAK and p-FAK on the basal and luminal surfaces of the mesencephalon under control and increased pressure conditions. (A-F) Changes in FAK expression; (G-L) changes in p-FAK expression. There is a pair of micrographs illustrating the changes that are shown in each of the bar graphs. The rectangles on each of the sections are the areas on the surfaces (basal/luminal) that were measured for gray values and used in the bar graphs. (A,B) FAK decreases 22\% (not statistically significant) on the basal surface when the mesencephalon is under increased pressure. (C,D) There is no change of FAK on the lumen surface when the brain is under pressure. In contrast, p-FAK exhibits a $50 \%$ decrease on the basal surface of the mesencephalon under pressure $\mathbf{( G , H , K ) ~ c o m p a r e d ~ w i t h ~ a ~ s i g n i f i c a n t ~} 26 \%$ increase in p-FAK on the luminal surface of mesencephalons under increased pressure $(\mathbf{I}, \mathbf{J}, \mathbf{L})$. 
positive pressure of CSF and neuroepithelium growth increases by cell proliferation. We have previously shown a correlation between an increase in intraluminal pressure and an increase in mitosis in the embryonic brain (Desmond et al., 2004); however, the link between the two has not been established as it has been for other physiological systems regulated by intraluminal pressure. Such a system is the aorta. The aorta has been studied extensively by Lehoux, et al., 2005. They have shown that an increase in intraluminal pressure increases the activation of FAK, Src, and Erk. When doubling the steady pressure in cultured aorta segments, Lehoux et al., 2003, 2005 showed via immunohistochemical localization that FAK phosphorylation was enhanced on the luminal surface, i.e. the endothelium plus juxtaposed layer of vascular smooth muscle cells. Additionally, lysates of aortas previously subjected to steady pressure exhibited three times the phosphorylated FAK and ERK1/2 relative to total immunoprecipitated FAK or Erk compared with controls. High intraluminal pressure $(150 \mathrm{~mm} \mathrm{Hg})$ activated FAK at several tyrosine residues: Tyr-397, Tyr-407, Tyr- 861 and Tyr925. The investigators further showed that FAK activation induced by steady high pressure was partially reduced in the presence of
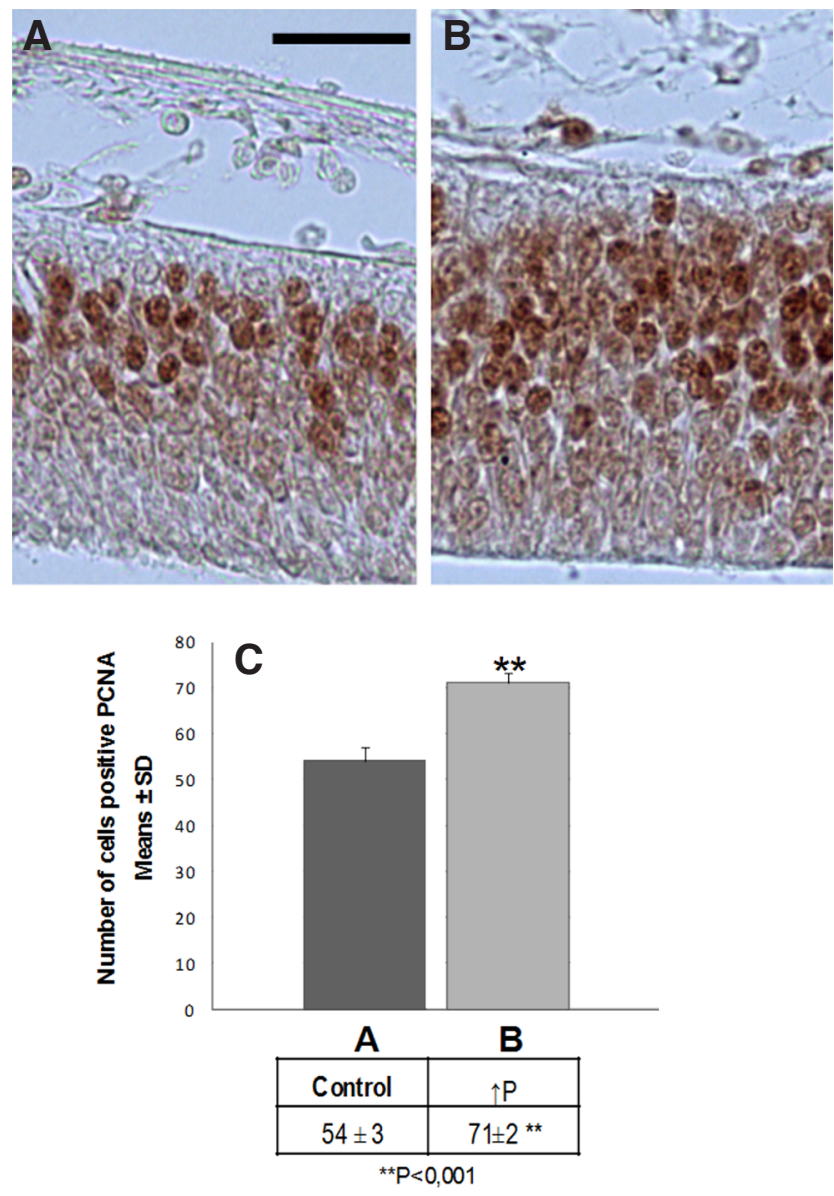

Fig. 6. The neuroepithelium exhibits a significant increase in PCNA labeled cells even when $\beta$-d-xyloside is in the culture medium sug-

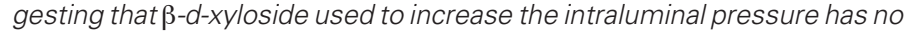
deleterious effects on normal metabolism of the cells. The photomicrographs are from untreated $(\mathbf{A})$ and treated embryos $(\mathbf{B})$ with $\beta$ - $d$-xyloside which is in our experiments to increase intraluminal pressure. The data includes 36 embryos. The magnification bar represents $15 \mu \mathrm{m}$. a Src family kinase inhibitor and that FAK coimmunoprecipitated with Grb2. Thus, they were also able to partially characterize the pathway of FAK activation induced by steady high pressure. Our results differed from Lehoux, et al., 2005 in that embryonic brains exposed to chronic high pressure showed a differential pattern of FAK activation but not an actual increase in the amount. One explanation for this difference in results may be because the high pressure we introduced in the embryonic brain was many fold less than what Lehoux, et al., introduced into the aorta.

FAK activation also has been exhibited by endothelial cells and vascular smooth muscle cells in culture conditions exposed to steady stretch (Yano, et al., 1996; Li, et al., 2003). These in vitro experiments parallel other studies using cultures of human pulmonary epithelial cells showing that Src and FAK mediate mechanical strain-induced cell proliferation and ERK1/2 phosphorylation (Chaturvedi, et al., 2007). This study with H144 pulmonary epithelial cells nicely correlates an increase in cell proliferation with an increase in phosphorylation of FAK, Src and ERK1/2 but doesn't demonstrate localization of FAK, either total or phosphorylated. The FAK residues that were activated by cyclic strain were tyr 397 and tyr 576.

\section{FAK expression in the neuroepithelium modified by intra- luminal pressure}

Two findings in our results support the idea that FAK activation is integral to brain growth. One is that p-FAK increases early on in brain growth, once the neural tube is closed as a result of occlusion of the spinal neurocoel and thus is under pressure (Desmond \& Schoenwolf, 1985). The other is that there is an increase in p-FAK on the luminal surface in response to an increased intraluminal pressure.

\section{FAK as a mechanotransducer of intraluminal pressure that promotes mitosis}

In other model systems, the relation between pressure and mitosis has been established as a receptor complex mostly identified as integrins associated with an intracellular cascade consisting of FAK, Src and Erk. The phosphorylation of these elements determine the degree of stimulation of mitosis. Integrins on the apical surface of the neuroepithelium have been previously described by others (Caprila, et al., 2009). In our study we show that FAK is present within neuroepithelial cells and exhibits a pattern of phosphorylation expression associated with closure of the neural tube and establishment of a closed pressure system (Desmond and Jacobson, 1977; Desmond, 1982). However, when we induced changes in the internal pressure exerted on the luminal surface, we didn't find changes in the amount of phosphorylation of FAK but we did find a relocalization of FAK activation to the luminal side from the basal side. We hypothesize that these mechanotransducers are the link between intra-luminal pressure and increased mitosis as shown in the model presented in Fig. 7.

Discovering FAK within the neuroepithelium is an extremely important finding because it has been known for years that growth of the vertebrate embryonic brain requires a delicate balance between increases of cavity size via fluid acquisition to maintain intraluminal pressure and increases in tissue volume via cellular proliferation (Desmond \& Jacobson 1977; Pacheco et al., 1986; Alonso et al., 1998, 1999; Desmond et al., 2005; Gato \& Desmond, 2009; Levitan \& Desmond, 2010). Perhaps this is better understood in light of 


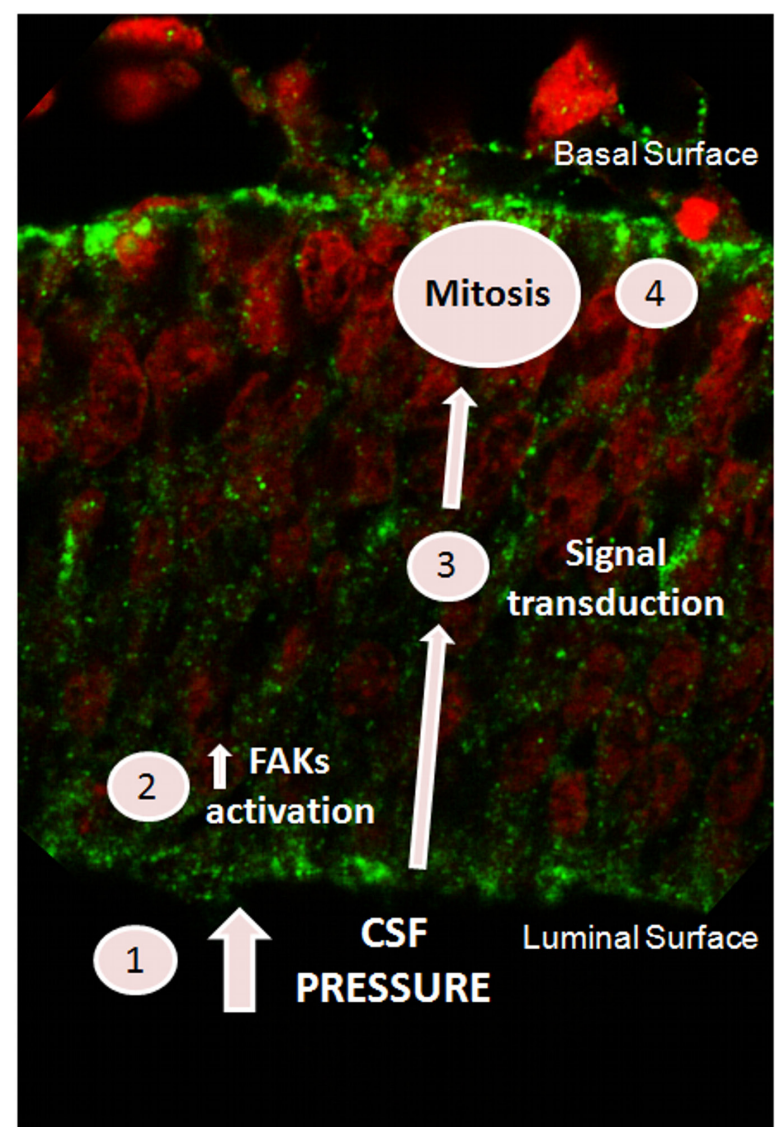

Fig. 7. A schematic summary of the response of FAKs to hyper pressure (1) within the embryonic brain cavity. There is a marked re-patterning of FAK activation (2) from the basal surface to the luminal surface. This phosphorylation of FAK (3) is correlated with an increase in cell proliferation (4) via mitosis.

the fact that the early vertebrate embryonic brain is mostly cavity $(70 \%)$ surrounded by a relatively thin neuroepithelium $(30 \%)$. Balance between the growth of the two components is required to maintain the normal architecture of the embryonic brain. Failure of the tissue to grow while the pressure increases often results in a thin neuroepithelium and a greatly expanded cavity common to hydrocephaly.

If the tissue grows at a rate greater and out of balance with the internal cavity pressure, the tissue folds into the cavity which is common in a defect known as the Chiari II malformation (McLone \& Knepper, 1989). Thus, failure to maintain balance between growth of the two interdependent systems results in many congential defects.

Although much is known about the rates of growth of the embryonic brain, the pressure generated by the accumulation of CSF within the cavity and the role of FGF-2 in stimulating neuron proliferation and differentiation, nothing is known about the molecular-cellular mechanism by which intraluminal pressure induces cell proliferation. This study shows for the first time that a known mechanotranducer, FAK, is active in the neuroepithelium during the period of rapid growth.

\section{FAK as a facilitator of neuronal cell alignment}

In addition to inducing mitosis, FAK may play another important role during growth of the neuroepithelium, namely, it may aid in in- tercalating the newly formed daughter cells into the neuroepithelium. The fact that we found p-FAK throughout the neuroepithelium as well as on the two surfaces suggests that $p$-FAK may aid in intercalation of the newly formed daughter cells resulting from mitosis. This may be facilitated by loosening of the membrane attachments to the extra-cellular matrix (ECM), which is also characteristic of cells prior to metastasis (Chalkiadalki et al., 2009). Several studies support the role of FAK as a modulator between cell adhesion to the ECM and proliferation as shown for endothelium (Ishida et al., 1996; Lehoux \& Tedgui 2003; Pirone et al., 2006). Focal adhesion disassembly prior to chemotaxis has not only implicated FAK but also FGF-2 via another non-receptor protein kinase, Fes (Kanda et al., 2006). These researchers showed that FGF-2 acted on Fes to modulate Src activation by FAK. We propose that this may also be true for the embryonic neuroepithelium for which we have shown previously that both intraluminal pressure and FGF-2 increase cell proliferation (Desmond et al., 2005; Gato et al., 2005; Martin et al., 2006).

Multiple mechanisms of regulating epithelial cell replication

Many studies have shown that there are receptors for growth factors on the luminal surface of the neuroepithelium (Parada, etal., 2008). These factors arise from within the neuroepithelial cells and the CSF (Lehtinen, et al., 2011). Here we describe a complementary mechanism to regulate embryonic brain growth. This mechanism most likely occurs during the period of rapid brain growth as an extra way to induce mitosis to accommodate the rapid growth.

\section{Perspectives on the pressure-FAK regulation system in brain growth}

This study emphasizes the importance of mechano-physical properties in morphogenesis. Despite the importance of molecular mechanisms, mechano-physical mechanisms are relevant to understand the complexity of embryonic development. Molecular functional experiments need to be done to test the hypothesis that $p-F A K$ is the essential mechanotransducer in the neuroepithelium that sends its signal to the nucleus via the MEK pathway. Future experiments need to explore the effect of specific inhibitors of FAK phosphorylation as well as the immunolocalization of Src and ERK during same period of rapid embryonic brain expansion.

\section{Materials and Methods}

\section{Cultivation and fixation of embryos}

Chick eggs from White Leghorn hens were incubated at $38^{\circ} \mathrm{C}$ and $98 \%$ relative humidity in a forced air incubator for the appropriate times to collect embryos for 12 Hamburger and Hamilton stages 8, 9,10,11, 12 , 13, 14,15, 18, 20, 21, 24 (Hamburger and Hamilton, 1955). These stages represent the period immediately preceding neural tube closure $(8,9,10)$, just after closure $(11,12)$ and the period of rapid brain enlargement following closure $(13,14,15,18,20,21,24)$. After excision from the yolk, the embryos were rinsed in physiological saline $(0.9 \%)$, blotted and fixed in Carnoy's fixative for one hr followed by dehydration in an ethanol series, clearing in xylene and embedding in Paraplast. Serial sections were cut at $8 \mu \mathrm{m}$, and transferred to 3-aminopropyl triethoxysilane (Sigma A3648) coated glass slides for immunolabeling. A minimum of three embryos per stage were serially sectioned and immunolabeled.

\section{Immunolocalization of FAK for confocal microscopy}

The sections were pre-blocked with freshly prepared $3 \%$ nonfat dry milk in PBS with $0.05 \%$ Tween-20 for 20 min at room temperature then 
incubated with a 1:1000 dilution of anti- phospho-FAK (tyr861 or tyr 397 -sources are in Table below) prepared in PBS-milk overnight at $4^{\circ} \mathrm{C}$. Next they were rinsed twice with distilled water before incubating with an Alexa 488-conjugated goat anti-rabbit IgG (Invitrogen A11008) at 1:5000 dilution in PBS-milk for $1.5 \mathrm{hr}$ at room temperature. After incubation, they were washed twice with distilled water, followed by washing in Tween-20 $(0.1 \%)$ for $3-5 \mathrm{~min}$ and five changes of distilled water before mounting with aqueous gel mount (Sigma G0918). Fluorescence images were obtained with a Leica TCS SPE confocal laser microscope equipped with a solid state laser with a $488 \mathrm{~nm}$ excitation filter and a dry $40 \mathrm{X}$ objective with a pinhole of 1 so that the optical slice thickness was $1.7 \mu \mathrm{m}$.

Controls for the specificity of antibody labeling were treated as above but without the primary antibody. Three antibodies were used for the immunostaining of brain sections; anti-FAK, anti-pFAK 397, and anti-pFAK 861. Because all three antibodies were produced in rabbits, the same conjugated anti-rabbit secondary was used to fluorescently label all of the sections. A negative control section exposed to the fluorescent secondary antibody but no primary antibody showed no signal. This control is valid for all three primary antibody probes.

\section{Western blot analysis of FAK and p-FAK}

Embryos (stages 10, 12, 14, 18-20, 24-25) were collected, the heads excised at the mesencephalo-rhombencephalic border and the eyes removed from all embryos stage 14 and older. The excised brains were flash frozen in liquid N2 and pooled on dry ice during the collection to prevent degradation. The tissue was homogenized mechanically in RIPA extractionlysis buffer (Fisher, \#89900) with protease and phosphatase inhibitors (Pierce Chemicals), by forcing though a 23 gauge needle. Lysates were then stored in liquid N2. The number of brains per homogenate varied per group. The brain sample was thawed and homogenized again by forcing through a 23 gauge needle just before loading and running the gel. A Bradford assay (Thermo Fisher Scientific 23225) was done to determine protein concentration. A volume of sample containing $10 \mu \mathrm{g}$ of protein supplemented with RIPA buffer (Thermo Fisher Scientific 89900) to $13 \mu \mathrm{L}$ and 2X Laemmeli buffer to $26 \mu \mathrm{L}$ total volume per sample was added to each well of a SDS-PAGE $10 \%$ gradient gel (BIO-RAD). The proteins were transferred to a PVDF membrane, the membrane was blocked with $5 \%$ nonfat dry milk and then incubated with diluted primary antibody (1:1500) at $4^{\circ} \mathrm{C}$ overnight followed by rinsing and incubation with the secondary antibody(1:5000) for one hr at RT. Next the membrane was washed in TBST and incubated with a 1:1 solution of luminal:peroxide Chemi-reagent (PIERCE West dura). The blotted membrane was then imaged on an Alpha Innotech Gel Documentation apparatus. The source and specific antibodies used for western detection and immunolocalization are:

\begin{tabular}{llll} 
Antibody Name & Supplier & AB No. & Target \\
\hline Anti-phospho-FAK, tyr 861861, polyclonal & Thermo Scientific & A1-03072 & p-FAK, tyr 861 \\
Anti-phospho-FAK, tyr 397, polyclonal & Thermo Scientific & PA5-17084 & p-FAK, tyr 397 \\
Anti- $\beta$-actin(N-21) & Santa Cruz Biotech & 130656 & B-actin \\
Anti-GAPDH monoclonal & Thermo Scientific & MA1-22670 & GAPDH \\
\hline
\end{tabular}

\section{Quantification of band intensities}

Western blots were imaged using an Alpha Innotech gel documentation system. Band intensities were quantified from these images using Image $\mathrm{J}$ gel analysis. For each time point, intensities for the detected band were normalized to the signal obtained for GAPDH from the same sample. Indicated values are presented with the maximum FAK/GAPDH ratio set to $100 \%$ (Fig. 3B).

\section{Pressure manipulation experiments}

Two day old chick embryos were placed into shell-less culture (Desmond \& Jacobson, 1977; Tuan 1980a), allowed to develop until stage 20 when $100 \mu$ of $4 \mathrm{mM} 4$-nitrophenyl - $\beta$-D- xylopyranoside (Sigma \#N2132) was injected subgerminally to increase the intraluminal pressure 3-5 fold based on earlier work (Alonso et al., 1993). We confirmed for this study that increasing the intraluminal pressure by increasing the osmolarity via 4-nitrophenyl- $\beta$-D- xylopyranoside (BDX) would also increase mitosis just as had been shown earlier by increasing pressure by adding fluid and keeping the osmolarity constant (Desmond et al., 2005). Based on analyzing PCNA (proliferating cell nuclear antigen; Chemicon, \#407) positive cells per area compared with controls for the neuroepithelia of four experimental and four control embryos, we found the increase in mitosis was $17 \%(71+$ vs 54+ 3 labeled; Fig. 6).

Embryos were then allowed to develop $43 \mathrm{~h}$ until they were stage 24 (Alonso et al., 1993). An equal number of untreated embryos were staged and incubated along with the treated embryos to control for incubator conditions. Both treated and control embryos were excised from the yolk and fixed immediately in Carnoys fluid for one hour. Then the embryos were prepared for serial sectioning as described earlier and prepared for immunolocalization of $p$ - FAK, and FAK as well as for western blot analysis. A minimum of three serially sectioned embryos for each treatment was analyzed and six excised brains were pooled for the western analysis.

\section{Analysis of fluorescence}

A semi-quantitative evaluation of fluorescence intensity was made by measuring the gray scale values for 10 representative images (1024×1024 pixels) for each condition: basal and apical surface regions of the mesencephalon from both control and increased pressure embryos. Areas of the brightness intensity were chosen for analysis. The fluorescence intensity of these areas was captured with a Leica confocal laser microscope TCP SPE with a $40 X$ objective excited at a wavelength of $488 \mathrm{~nm}$. The software used for the quantification of fluorescence was Leica LAS AF 2.3.6. In all cases, the slice thickness was uniform as were the conditions of brightness and contrast and intensity of the laser itself. In each case a standard rectangular region of interest (33,000 pixels) was recorded as gray scale values.

\section{Organotypic neuroepithelial cell culture}

CSF was removed from control embryos and BDX treated embryos and then applied to excised mesencephalic neuroepithelium in organotypic culture as previously described (Gato, et al., 2005). Epithelial cells undergoing DNA synthesis were identified by PCNA immunolabeling.

\section{Acknowledgements}

MED gratefully acknowledges the financial support of the Department of Biology and Villanova University for a sabbatical leave in order to complete this study. AG is grateful for support from Ministerio de Educacion y Cinencia BFU/6516 and Juntal de Castillo y Leon GR 195. All authors acknowledge the contribution of masters student, Laura Figueroa to provide the PCNA data. Electrophoretic data presented in this paper represent materials submitted by E.M. in partial fulfillment of the degree of Master of Science in Biology, Villanova University.

\section{References}

ALONSO, M.I., GATO, A., MORO, J.A. and BARBOSA, E. (1998). Disruption of proteoglycans in neural tube fluid by beta-D-xyloside alters brain enlargement in chick embryos. Anat Rec. 252: 499-508.

ALONSO, M.I., GATO, A., MORO, J.A., MARTIN, P. and BARBOSA, E. (1999). Involvement of sulfated proteoglycans in embryonic brain expansion at earliest stages of development in rat embryos. Cells Tissues Organs. 165: 1-9.

BANG, O.S., KIM, E.J., CHUNG, J.G., LEE, S.R., PARK, T.K. and KANG, S.S. (2000). Association of focal adhesion kinase with fibronectin and paxillin is required for precartilage condensation of chick mesenchymal cells. Biochem Biophys Res Commun. 278: 522-529.

BOUTAHAR, N., GUIGNANADON, A., VICO-, L. and LAFAGE-PROUST, M.H. (2004). Mechanical strain on osteoblasts activates autophosphorylation of focal adhesion kinase and proline-rich tyrosine kinase 2 tyrosine sites involved in ERK activation. J Biol Chem. 279: 30588-30599.

BRAREN, R., HU, H., KIM, Y.H., BEGGS, H.E., REICHARDT, L.F. and WANG, R. (2006). Endothelial FAK is essential for vascular network stability, cell survival, and lamellipodial formation. J Cell Biol. 172: 151-162. 
CAI, W.J., LI, M.B., WU, X., WU, S., ZHU, W., CHEN, D., LUO, M., EITENMULLER, I., KAMPMANN, A., SCHAPER, J. and SCHAPER, W. (2009). Activation of the integrins alpha 5 beta 1 and alpha $v$ beta 3 and focal adhesion kinase (FAK) during arteriogenesis. Mol Cell Biochem. 322: 161-169.

CALALB, M.B., ZHANG, X., POLTE, T.R. and HANKS, S.K. (1996). Focal adhesion kinase tyrosine-861 is a major site of phosphorylation by Src. Biochem Biophys Res Commun. 228: 662-668.

CAPTILE, T., OSORIO, G., HENRIQUEZ, J.P. and MONTECINOS, H. (2009). Polarized expression of integrin beta1 in diencephalic roof plate during chick development, a possible receptor for SCO-spondin. Dev Dyn. 238: 2494-2504.

CHALKIADAKI, G., NIKITOVIC, D., BERDIAKIi, A., SIFAKI, M., KRASAGAKIS, K., KATONIS, P., KARAMANOS, N.K. and TZANAKAKIS, G.N. (2009). 2007 Fibroblast growth factor-2 modulates melanoma adhesion and migration through a syndecan-4- dependent mechanism. Int J Biochem Cell Biol. 41: 1323-1331.

CHATURVEDI, L.S., MARSH, H.M. and BASSON, M.D. (2007). Src and focal adhesion kinase mediate mechanical strain-induced proliferation and ERK1/2 phosphorylation in human $\mathrm{H} 441$ pulmonary epithelial cells. Am J Physiol Cell Physiol. 292: C1701-C1713.

CHAZIZACHARIAS, N.A., KOURAKLIS, G.P. and THEOCHARIS, S.E. (2010). The role of focal adhesion kinase in early development. Histol Histopathol. 25: 1039-1055.

CHO, H.M., CHOI, S.H., HWANG, K.C., OH, S.Y., KIM, H.G., YOON, D.H., CHOI, M.A., LIM, S., SONG, H., JANG, Y. and KIM, T.W. (2005). The SrC/PLC/PKC/ MEK/ERK signaling pathway is involved in aortic smooth muscle cell proliferation induced by glycated LDL. Mol Cells. 19: 60-66.

DESMOND, M.E. (1985). Reduced number of brain cells in so-called neural overgrowth. Anat Rec. 212: 195-198.

DESMOND, M.E. and JACOBSON, A.G. (1977). Embryonic brain enlargement requires cerebrospinal fluid pressure. Dev Biol. 57: 188-198.

DESMOND, M.E. and LEVITAN, M.L. (2002). Brain expansion in the chick embryo initiated by experimentally produced occlusion of the spinal neurocoel. Anat Rec. 268: 147-159.

DESMOND, M.E., LEVITAN, M.L. and HAAS, A.R. (2005). Internal luminal pressure during early chick embryonic brain growth: descriptive and empirical observations. Anat Rec A Discov Mol Cell Evol Biol. 285: 737-747.

DESMOND, M.E. and SCHOENWOLF, G.C. (1985). Timing and positioning of occlusion of the spinal neurocele in the chick embryo. J Comp Neurol. 235: 479-487.

EVANS, A.L. and MULLER, U. (2000). Stereocilia defects in the sensory hair cells of the inner ear in mice deficient in integrin $\alpha 8 \beta 1$. Nature genetics. 24: 424-428.

GALBRAITH, C.G., YAMADA, K.M. and SHEETZ, M.P. (2002). The relationship between force and focal complex development. J Cell Biol. 159: 695-705.

GATO, A. and DESMOND, M.E. (2009). Why the embryo still matters: CSF and the neuroepithelium as interdependent regulators of embryonic brain growth, morphogenesis and histiogenesis. Dev Biol. 327: 263-272.

GATO, A., MORO, J.A., ALONSO, M.I., BUENO, D., DE LA MANO, A. and MARTIN, C. (2005). Embryonic cerebrospinal fluid regulates neuroepithelial survival, proliferation, and neurogenesis in chick embryos. Anat Rec A Discov Mol Cell Evol Biol. 284: 475-484.

GATO, A., MORO, J.A., ALONSO, M.I., PASTOR, J.F., REPRESA, J.J. and BARBOSA, E. (1993). Chondroitin sulphate proteoglycan and embryonic brain enlargement in the chick. Anat Embryol (Berl). 188: 101-106.

GIRAULT, J.A., COSTA, A., DERKINDEREN, P., STIDLER, J.M. and TOUTANT, M. (1999). FAK and PYK2/CAKbeta in the nervous system: a link between neuronal activity, plasticity and survival? Trends Neurosci. 22: 257-263.

HAMBURGER, V. and HAMILTON, H.L. (1951). A Series of Normal Stages in the Development of the Chick Embryo. J. Morphology. 88: 49-92.

HENS, M.D. and DESIMONE, D.W. (1995). Molecular Analysis and Developmental Expression of the Focal Adhesion Kinase Pp125(Fak) in Xenopus-Laevis. Dev Biol. 170: 274-288.
ISHIDA, T., PETERSON, T.E., KOVACH, N.L. and BERK, B.C. (1996). MAP kinase activation by flow in endothelial cells - Role of beta 1 integrins and tyrosine kinases. Circ. Res. 79: 310-316.

KANDA, S., MIYATA, Y., KANETAKE, H. and SMITHGALL, T.E. (2006). Fibroblast growth factor-2 induces the activation of Src through Fes, which regulates focal adhesion disassembly. Exp. Cell Res. 312: 3015-3022.

KATSUMI, A., NAOE, T., MATSUSHITA, T., KAIBUCHI, K. and SCHWARTZ, M.A. (2005). Integrin activation and matrix binding mediate cellular responses to mechanical stretch. J. Biol. Chem. 280: 16546-16549.

KOKKINOS, M.I., BROWN, H.J. and DE IONGH, R.U. (2007). Focal adhesion kinase (FAK) expression and activation during lens development. Molec. Vis. 13:418-430.

LAEMMLI, U.K. (1970). Cleavage of structural proteins during the assembly of the head of bacteriophage T4. Nature. 227: 680-685.

LEHOUX, S., ESPOSITO, B., MERVAL, R. and TEDGUI, A. (2005). Differential regulation of vascular focal adhesion kinase by steady stretch and pulsatility. Circulation. 111: 643-649.

LEHOUX, S. and TEDGUI, A. (2002). Cellular mechanics and gene expression in blood vessels. J Biomechanics. 36: 631-643.

LEHTINEN, M.K., ZAPPATERRA, M.W., CHEN, X., YANG, Y.J., HILL, A.D., LUN, M. MAYNARD, T., GONZALEZ, D., KIM, S., Ye, P., D’ERCOLE, A.J., WONG, E.T., LAMANTIA, A.S. and WALSH, C.A. (2011) The cerebrospinal fluid provides a proliferative niche for neural progenitor cells. Neuron. 69: 893-905

LEVITAN, M.L. and DESMOND, M.E. (2009). Expansion of the human embryonic brain during rapid growth: area analysis. Anat Rec (Hoboken). 292: 472-480.

LI, C., WERNIG, F., LEITGES, M., HU, Y. and XU, Q. (2003). Mechanical stress-activated PKCdelta regulates smooth muscle cell migration. FASEB J. 17: 2106-2108.

MARTIN, C., BUENO, D., ALONSO, M.I., MORO, J.A., CALLEJO, S., PARADA, C MARTIN, P., CARNICERO, E. and GATO, A. (2006). FGF2 plays a key role in embryonic cerebrospinal fluid trophic properties over chick embryo neuroepithelial stem cells. Dev Biol. 297: 402-416.

MCLONE, D.G. and KNEPPER, P.A. (1989). The cause of Chiari II malformation: a unified theory. Pediatr Neurosci. 15: 1-12.

MITRA, S.K., HANSON, D.A. and SCHLAEPFER, D.D. (2005). Focal adhesion kinase: in command and control of cell motility. Nat Rev Mol Cell Biol. 6: 56-68.

PACHECO, M.A., MARKS,D. R.W., SCHOENWOLF, G.C. and DESMOND, M.E. (1986). Quantification of the initial phases of rapid brain enlargement in the chick embryo. Am J Anat. 175: 403-411.

PARADA, C., GATO, A. and BUENO, D. (2008). All-trans retinol and retinol-binding protein from embryonic cerebrospinal fluid exhibit dynamic behaviour during early central nervous system development. Neuroreport. 19: 945-950.

PIRONE, D.M., LIU, W.F., RUIZ, S.A., GAO, L., RAGHAVEN, S., LEMMON, C.A., ROMER, L.H. and CHEN, C.S. (2006). An inhibitory role for FAK in regulating proliferation: a link between limited adhesion and RhoA-ROCK signaling. J Cell Biol. 174: 277-288

SCHWARTZ, M.A. (2001). Integrin signaling revisited. Trends Cell Biol. 11: 466-470

SUSAKI, K. and CHIBA, C. (2007). MEK mediates in vitro neural transdifferentiation of the adult newt retinal pigment epithelium cells: Is FGF2 an induction factor? Pigment CellRes. 20: 364-379.

TUAN, R.S. (1980). Calcium transport and related functions in the chorioallantoic membrane of cultured shell-less chick embryos. Dev Biol. 74: 196-204.

YANO, Y., GEIBEL, J. and SUMPIO, B.E. (1996). Tyrosine phosphorylation of pp125FAK and paxillin in aortic endothelial cells induced by mechanical strain Am J Physiol. 271: C635-C649.

XIAO, T., SALGIA, R., LI, J.-L., GRIFFIN, J.D. and HOWLEY, P.M. (1997). The bovine papillomavirus E6 protein binds to the LD motif repeats of paxillin and blocks its interaction with vinculin and the focal adhesion kinase. J. Biol. Chem 272: 33373-33376

ZHAO, X. and GUAN, J.L. (2011). Focal adhesion kinase and its signaling pathways in cell migration and angiogenesis. Adv Drug Deliv Rev. 63: 610-615. 


\section{Further Related Reading, published previously in the Int. J. Dev. Biol.}

Cell cycle control of Notch signaling and the functional regionalization of the neuroepithelium during vertebrate neurogenesis Maria Jesús Latasa, Elsa Cisneros and Jose María Frade

Int. J. Dev. Biol. (2009) 53: 895-908

The surface ectoderm of the chick embryo exhibits dynamic variation in its response to neurogenic signals

Vineeta-Bhasker Tripathi, Yasuo Ishii, Muhammad M. Abu-Elmagd and Paul J. Scotting Int. J. Dev. Biol. (2009) 53: 1023-1033

Cellular dynamics and molecular control of the development of organizer-derived cells in quail-chick chimeras

Jean-Baptiste Charrier, Martin Catala, Françoise Lapointe, Nicole Le Douarin and MarieAimée Teillet

Int. J. Dev. Biol. (2005) 49: 181-191

The choice between epidermal and neural fate: a matter of calcium.

Marc Moreau and Catherine Leclerc

Int. J. Dev. Biol. (2004) 48: 75-84

Genetic and epigenetic control of midbrain dopaminergic neuron development. C Perrone-Capano and U Di Porzio Int. J. Dev. Biol. (2000) 44: 679-687

5 yr ISI Impact Factor $(2011)=2.959$

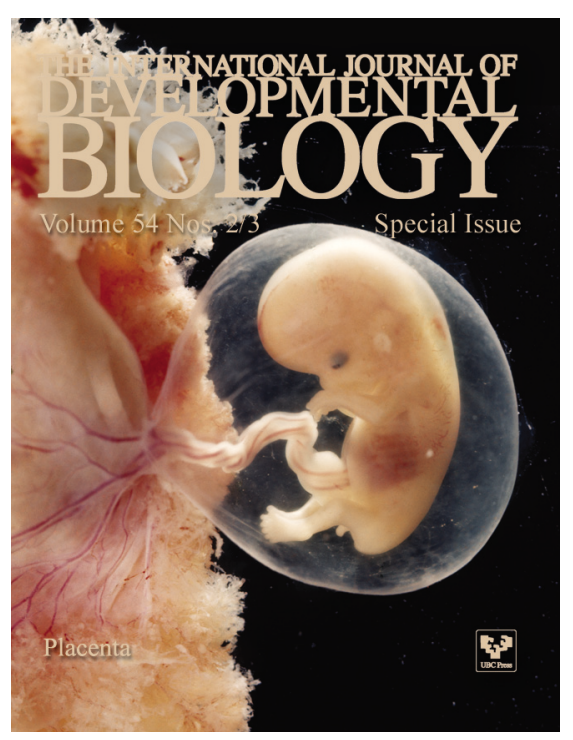

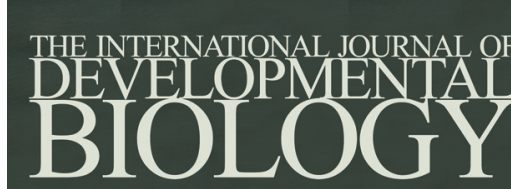

Volume 55 Nos. $4 / 5$

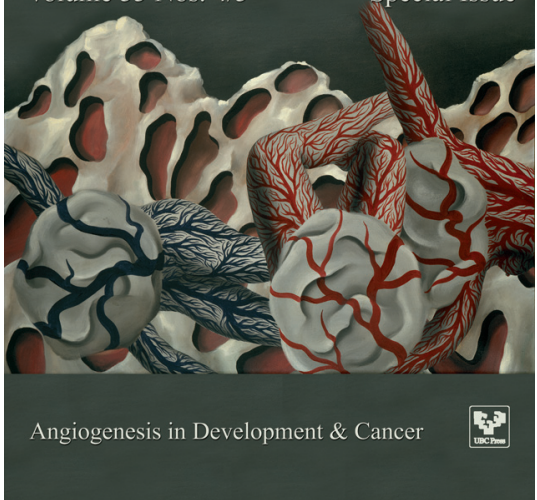

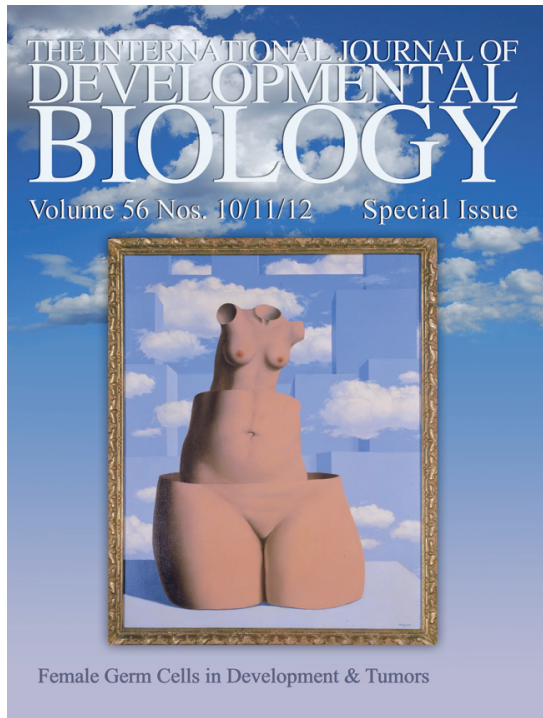

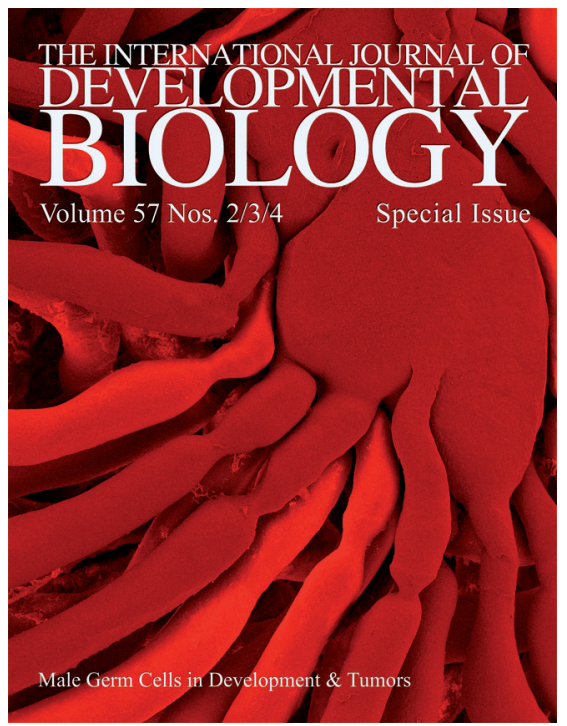

